\title{
Investigation of germanium implanted with hydrogen for layer transfer applications
}

\author{
T.S. Perova ${ }^{1}$, B.M. Armstrong ${ }^{2}$, J. Wasyluk ${ }^{1}$, P.Baine ${ }^{2}$, P. Rainey ${ }^{3}$, \\ S.J.N. Mitchell ${ }^{2}$, D.W. McNeill ${ }^{2}$, H.S. Gamble ${ }^{2}$ and R. Hurley ${ }^{2}$ \\ ${ }^{1}$ Department of Electronic and Electrical Engineering, University of Dublin, Trinity College, Ireland \\ ${ }^{2}$ School of Electrical Engineering, Electronics and Computer Science, Queen's University Belfast \\ ${ }^{3}$ Biomedical Diagnostics Institute, Dublin City University, Ireland \\ perovat@tcd.ie,b.armstrong@ee.qub.ac.uk, wasyluj@tcd.ie,p.baine@ee.qub.ac.uk, \\ paul.rainey@dcu.ie,n.mitchell@ee.qub.ac.uk, dw.mcneill@ee.qub.ac.uk, \\ h.gamble@ee.qub.ac.uk, rhurley@vacuumtunes.co.uk
}

Keywords: Germanium on Insulator, MOSFETs, Ion cut, layer transfer, Spreading Resistance Profiling, micro-Raman Spectroscopy

\begin{abstract}
The technology for thin Ge layer transfer by hydrogen ion-cut process is characterised in this work. Experiments were carried out to determine suitable hydrogen ion implantation doses in germanium for the low temperature ion cut process by examining the formation of blisters on implanted samples. Raman and Spreading Resistance Profiling (SRP) have been used to analyse defects in germanium caused by hydrogen implants. Bevelling has been used to facilitate probing beyond the laser penetration depth. Results of Raman mapping along the projection area reveal that after post implant annealing at $400{ }^{\circ} \mathrm{C}$, some crystal damage remains, while at $600{ }^{\circ} \mathrm{C}$, the crystal damage has been repaired. SRP shows that some amount of hydrogen acceptor states $\left(\sim 1 \times 10^{16}\right.$ acceptors $\left./ \mathrm{cm}^{2}\right)$ remain after $600{ }^{\circ} \mathrm{C}$. These are thought to be vacancy-related point defect clusters.
\end{abstract}

\section{Introduction}

The main driving force for the future development of CMOS integrated circuits are high performance and system on a chip (SOC). Silicon on insulator (SOI) and germanium on insulator (GeOI) technologies offer reduced parasitic capacitance and process simplification. The reduced parasitic capacitance yields potential higher switching speed and/or reduced power dissipation in CMOS integrated circuits. Both technologies offer potential solutions suited to SOC. Germanium offers higher carrier mobility than silicon. As the scaling down of dimensions in IC technology approaches its limit, there is therefore an interest in $\mathrm{Ge}$ as an alternative semiconductor material potentially yielding superior high speed performance compared to silicon. The dominant technology to produce SOI employs wafer bonding. Thin silicon is a requirement and this has been achieved by the Smart $\mathrm{Cut}^{\odot}$ process [1], where hydrogen ion implantation is employed to yield sub-micron layer transfer. The technology with silicon is mature. Similar technology is being employed to transfer thin germanium layers. In order to exploit the performance advantages offered by Ge, it is vitally important that the transferred layer has high crystalline quality approaching that of bulk Ge. The technology for thin Ge layer transfer by hydrogen ion-cut process is characterised in this work. Pchannel MOSFETs are manufactured in GeOI made by ion-cut and by alternative standard techniques. The transistor properties are shown to be strongly dependent on the manufacturing technology employed for the thin layer production. The transferred layers have therefore been characterised by Spreading Resistance and Raman Spectrometry profiling of bevelled samples. This permits depth profiling for crystal defects over distances greater than the laser penetration. The results achieved provide an insight into serious issues for Ge layer transfer. 


\section{Results and Discussions}

Layer transfer technology. The starting material for MOS transistor manufacture is thin germanium on insulator. GeOI usually consists of a germanium layer on an oxidized silicon handle wafer. Alternatively the germanium layer can be on a sapphire substrate yielding GeOS, as in this work. Low temperature wafer bonding is employed to join the germanium wafer to the handle wafer. This technology has been described elsewhere [2]. In order to produce a thin Ge layer, the wafer is ion implanted with hydrogen. This is the Smart Cut ${ }^{\odot}$ or ion cut process which is a mature technology for silicon layer transfer. In order to establish the technology for Ge layer transfer onto either oxidized silicon or sapphire, experiments were carried out to determine suitable anneal schedules for a low temperature hydrogen ion-cut process. This was undertaken by examining the formation of blisters on implanted samples. As illustrated in Figure 1, in an ion cut process the bonded handle wafer acts as a stiffener, so that the crack created by the implanted hydrogen propagates laterally, resulting in the splitting or cutting of the original wafer and the transfer of a Ge layer to the handle wafer. The split occurs at the ion implantation range, and sub-micron layer thickness can be achieved with nanometer scale roughness which can be removed by touch polishing. Without this rigid support, surface blisters form, and it is known that there is a good correlation between the thermal budget required for blister formation and ion cut.
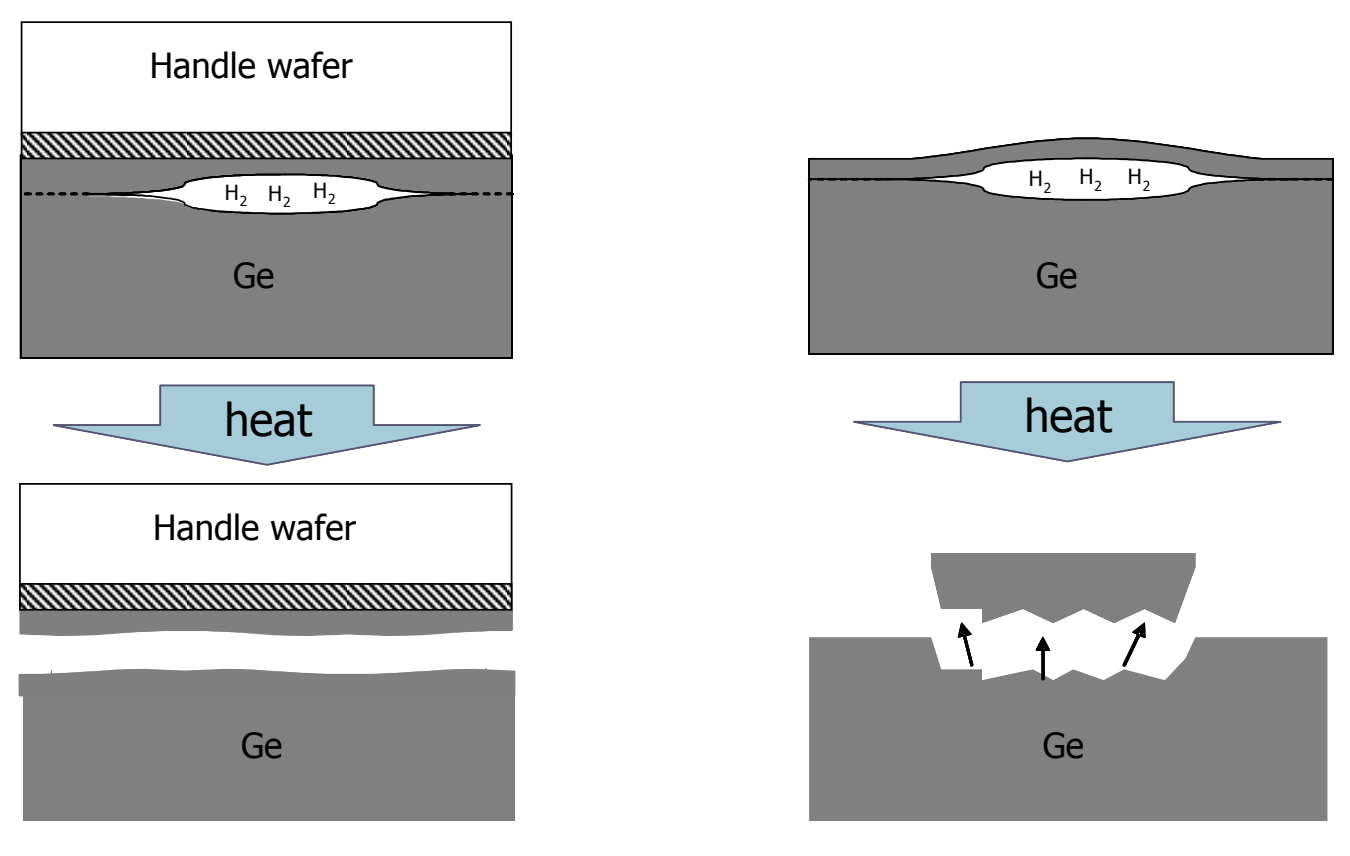

Figure 1. Cross-sectional schematics of (a) LAYER TRANSFER: lateral propagation of hydrogen filled cavities to achieve uniform delamination and (b) BLISTERING: in the absence of a stiffening handle wafer, the ripening and eventual local delamination of 4-10 $\mu \mathrm{m}$ diameter regions.

Blister tests were undertaken on germanium using an implantation energy of $120 \mathrm{keV}$ and an $\mathrm{H}^{+}$ion dose of $6 \times 10^{16} \mathrm{~cm}^{-2}$. Each sample was annealed at a fixed temperature in the range $250^{\circ} \mathrm{C}$ to $400^{\circ} \mathrm{C}$. The anneal time was carefully controlled and the samples were inspected using optical microscopy at various controlled time intervals until blistering of the surface was observed. Figure 2 shows an Arrhenius plot of time to blister at each temperature. The results for germanium are compared with similar blister formation data obtained for silicon. It can be observed that blistering in germanium takes shorter time than in silicon or alternatively that for a fixed time process it is possible to use a 
lower temperature anneal than that used for silicon. The anneal temperature for layer transfer to silicon handles is usually limited to an upper value of $300^{\circ} \mathrm{C}$ in order to avoid problems due to differences in thermal coefficient of expansion. Anneal time to achieve splitting and layer transfer is therefore approximately 30 hours. Transfer to sapphire can be done at higher temperature e.g. a 30 minute anneal at $350^{\circ} \mathrm{C}$.

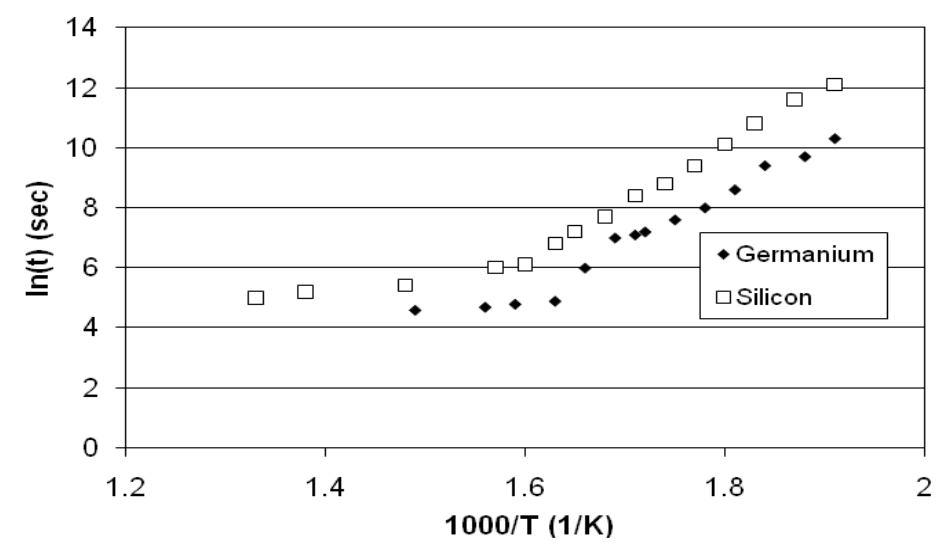

Figure 2. Aarhenius plot $\ln$ (time) against 1000/T for Ge and $\mathrm{Si}$ annealed under similar conditions

MOS Transistor manufacture. GeOS substrates have been employed in the manufacture of circular geometry p-channel MOS transistors. A self-aligned tungsten gate manufacturing process was employed. This had previously been developed and described for MOST manufacture on bulk Ge substrates [3]. Two types of GeOS substrates were employed (i) manufactured using H ion-cut technology and (ii) manufactured by standard grind and polish technology. The latter technology lacks the control of Ge layer thickness provided by the ion-cut process, and has been used here as a control reference. The output characteristics of the transistors are shown in Figure 3.

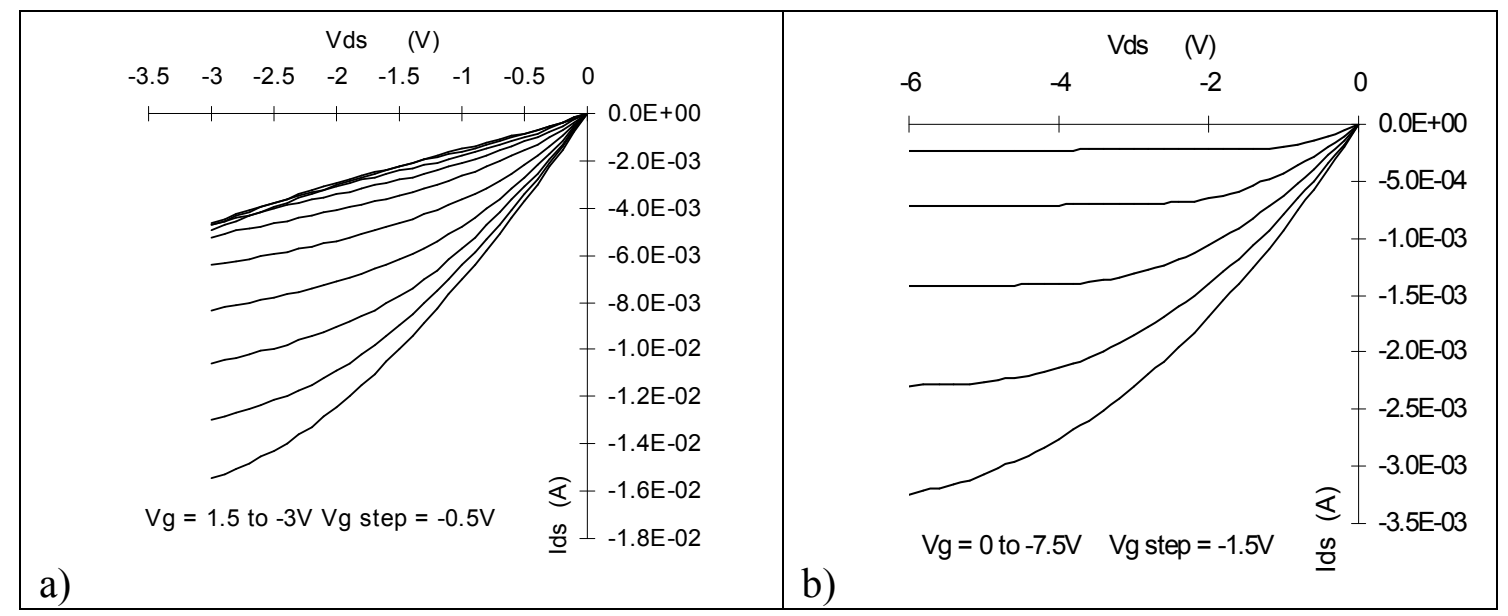

Figure 3. p-channel MOST output characteristics manufactured (a) on an ion cut substrate and (b) on a substrate thinned using grind and polish technology

The transistor characteristic of Figure 3 (a) exhibits a normally-on characteristic. The transistor cannot be switched off even by positive gate bias voltages. This is typical of all transistors manufactured on this ion-cut substrate. The transistors manufactured on the GeOS with no ion-cut process exhibit excellent characteristics as shown in Figure 3(b). The transistor can be switched off 
and has a threshold voltage of $-1 \mathrm{~V}$. Both transistors have been manufactured by identical process flows and employ Ge wafers of the same resistivity. The electrical characteristics indicate that the $\mathrm{Ge}$ in the ion-cut GeOS is $p$-type in the transistor channel. This has been investigated using Spreading Resistance Profiling (SRP) and Raman Spectroscopy.

Electrical and optical characterizations. The implanted Ge layers and completed GeOS samples were characterized for structural quality, stress, film uniformity, resistivity and carrier concentration, using optical microscopy, micro-Raman spectroscopy and SRP technique. Bevelling is routinely used in preparation of samples for SRP and can be useful in Raman spectroscopy analysis, where there is a requirement to probe deep into materials. Germanium substrates used in this study were $n$-type, Sb-doped and $p$-type, Ga-doped $<100>$ wafers with varying resistivities. Substrates were implanted with various fluences of hydrogen (ranging from 3 to $7 \times 10^{16} \mathrm{~cm}^{-2}$ ) with implantation energy of $75 \mathrm{KeV}$ selected for a projected range of around $350 \mathrm{~nm}$ into the germanium substrate. Substrates were aligned at a $7^{\circ}$ angle and kept at close to ambient temperature throughout the implant. Samples were beveled at Solecon Laboratories for SRP analysis (see [4,5] for details). Spreading resistance profiles were used to calculate carrier concentrations, based on the assumption that the mobilities of the samples are similar to calibration standards. The same samples were subsequently used for Raman analysis.

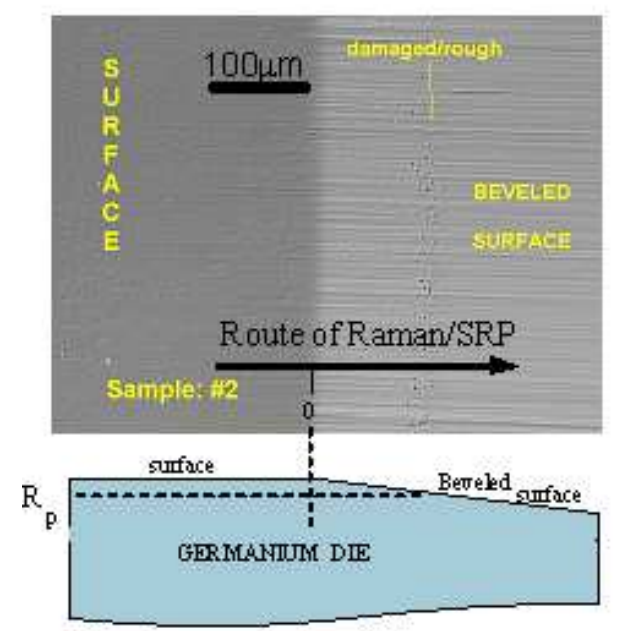

Figure 4. Plan view optical microscopy image of Ge sample (with schematic cross-section) implanted with $3 \times 10^{16}$ atoms $/ \mathrm{cm}^{2}$ hydrogen dose and annealed at $450{ }^{\circ} \mathrm{C}$. The arrow indicates the direction and distance of Raman line mapping.

Raman spectra were registered in backscattering geometry using a micro-Raman Renishaw 1000 system with $633 \mathrm{~nm}$ line of a HeNe laser as excitation source [5]. The laser spot of $\sim 2 \mu \mathrm{m}$ was focused on the sample surface using a 50x objective with a short-focus working distance. Line mapping measurements were performed at a distance along the bevelling surface ranging from 0 to $\sim 400 \mu \mathrm{m}$ with a step size of few microns, where zero corresponds to the starting point of the measurements (see Fig. 4).

Figure 4 shows an optical microscope image of a bevelled Ge sample implanted with $3 \times 10^{16}$ atoms $/ \mathrm{cm}^{2}$ hydrogen dose and annealed at $450{ }^{\circ} \mathrm{C}$. The structural damage caused by implanted hydrogen at the projected range is clearly observed in Figure 4. This dose of $3 \times 10^{16}$ atoms $/ \mathrm{cm}^{2}$ is slightly below the threshold of blistering for germanium since this is beneficial for analysis. Both SRP and Raman analysis would be detrimentally affected by the roughening caused by blisters. Bevelling reveals that some defects and cavities form at the projected range, due to clustering of molecular hydrogen, but these are not large enough to be visible at the surface. Raman spectra measured at the projection range for different Ge samples exhibit the Ge-Ge longitudinal optical (LO) phonon peak at $\sim 300 \mathrm{~cm}^{-1}$. The linewidth and intensity of Ge-Ge Raman peak are indicators of crystalline quality of the structure. The linewidth (or full width at half maximum, FWHM) 
increases and peak intensity decreases as the number of implant-related defects increase. When an implanted sample is annealed at high temperatures, the linewidth of the Ge-Ge peak returns to the value of high-crystalline-quality Ge.

In Figure 5 (a), the carrier concentration derived from resistivity, and Raman linewidth maps are plotted versus depth into a p-type Ge sample implanted with $6 \times 10^{16}$ atoms $/ \mathrm{cm}^{2}$ of $\mathrm{H}^{+}$, and without any subsequent anneal. Raman shows a $7 \mathrm{~cm}^{-1}$ linewidth peak, at a depth corresponding to the projected range. The resistivity $\rho$ measured at points along the bevel, is a function of the majority carrier concentration, $N$, and majority carrier mobility $\mu,[\rho=1 /(N q \mu)]$. Defects that impair crystal quality can cause a reduction in mobility, and therefore an increase in resistivity. The apparent drop in acceptor concentration in the initial $0.7 \mu \mathrm{m}$ depth is attributed to un-annealed implant damage, leading to mobility reduction.

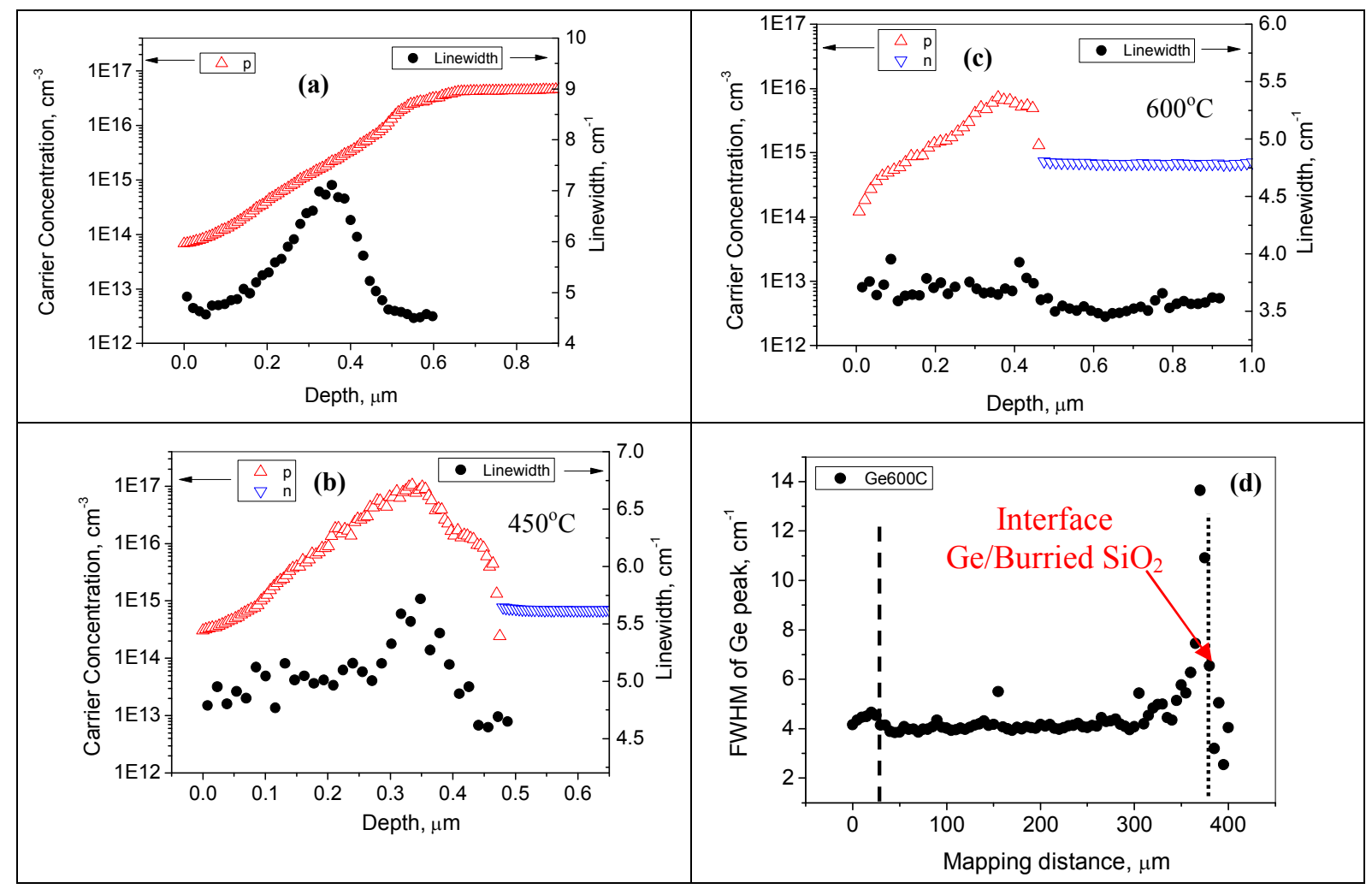

Figure 5. Raman linewidth and carrier concentration derived from SRP vs. depth into Ge sample implanted with (a) $6 \times 10^{16}$ atoms $/ \mathrm{cm}^{2} \mathrm{H}^{+}$, (b) $3 \times 10^{16}$ atoms $/ \mathrm{cm}^{2} \mathrm{H}^{+}$annealed to $450{ }^{\circ} \mathrm{C}$, and (c) to $600{ }^{\circ} \mathrm{C}$, (d) GeOS substrate with Ge layer implanted with $7 \times 10^{16}$ atoms $/ \mathrm{cm}^{2} \mathrm{H}^{+}$and annealed at 600 ${ }^{\circ} \mathrm{C}$.

In Figure 5(b), the depth profiles of the Raman linewidth and carrier concentration are presented for an $\mathrm{n}$ type sample implanted with $3 \times 10^{16}$ atoms $/ \mathrm{cm}^{2}$ of $\mathrm{H}^{+}$and annealed at $450{ }^{\circ} \mathrm{C}$. This is a dose slightly below the threshold of blistering for germanium. Note the carrier type change from $n$ to $p$. It is known that both hydrogen and point defects in Ge can act as acceptor states and both may be contributing to the acceptor profile after the $450^{\circ} \mathrm{C}$ anneal. The peak of $\sim 1 \times 10^{17} \mathrm{~cm}^{-3}$ acceptors and the maximum linewidth of Ge-Ge peak $\left(\sim 5.8 \mathrm{~cm}^{-1}\right)$ is observed at the projected range of $350 \mathrm{~nm}$. The projected range was confirmed by a SRIM [6] simulation, and by surface profile measurements on blistered samples and on layers which were transferred to handle substrates by smart-cut ${ }^{\odot}$. The Raman linewidth peak of $5.8 \mathrm{~cm}^{-1}$ detected at the projected range suggests some crystal disorder remaining after this anneal. For the $600{ }^{\circ} \mathrm{C}$ annealed sample (Fig. 5 (c)), SRP shows that the peak carrier concentration decreases about one order to $1 \times 10^{16}$ acceptors $/ \mathrm{cm}^{3}$, while the Raman linewidth profile is flat and corresponds to the high crystalline quality region of the un-implanted 
Ge wafer. This residual acceptor profile would act as a p-channel in the MOS transistor resulting in the normally on characteristic reported in Figure 3(a). As the atomic density of pure Ge is 4.42 $\times 10^{22}$ atoms $/ \mathrm{cm}^{3}$, this number of acceptors would equate to 1 defect in $4 \times 10^{6}$ atoms, approaching the detection limit for Raman measurements at this resolution. There is good correlation between the SRP and Raman at the deeper end of the implant (around $0.5 \mu \mathrm{m}$ deep).

The Raman mapping technique was also applied for the analysis of Ge-on-Sapphire wafers for the investigation of defects developed at the interfaces. The results of Raman mapping obtained for GeOS substrates after annealing at $600{ }^{\circ} \mathrm{C}$ is shown in Fig. 5 (d). Dashed and dotted lines correspond to $\mathrm{SiO}_{2} / \mathrm{Ge}$ and $\mathrm{Ge} /$ burried-SiO 2 interfaces, respectively. As can be seen from Fig. 5(d) the linewidth in thin Ge layer is around $4 \mathrm{~cm}^{-1}$ along the Raman pass up to $\sim 320 \mu \mathrm{m}$ mapping distance, which indicates good crystalline quality of $\mathrm{Ge}$ in this area. However, at the distance from 320 to $375 \mu \mathrm{m}$ a significant increase of FWHM is observed. Raman spectra, registered in this area, show a strong asymmetry from the low frequency part of the spectra, which assigned to the amorphous Ge. The investigation of the Ge samples with $\mathrm{SiO}_{2}$ capping layer, subjected subsequent hydrogen implantation and annealing, show no amorphization of $\mathrm{Ge}$ at the $\mathrm{Ge} / \mathrm{SiO}_{2}$ interface. We believe that disorder of the Ge lattice at the interface can be introduces at the stage of the layer transfer when implanted Ge is bonded to the sapphire substrate.

\section{Conclusion}

A comparison and correlation of the electrical and structural properties of implant-related defects has been performed for "smart-cut ${ }^{\odot}$ type" doses of hydrogen in germanium. Using SRP, it has been found that, for hydrogen implant, a quantity of $1 \times 10^{16} \mathrm{~cm}^{-2}$ electrically active acceptors still remain after annealing at $600^{\circ} \mathrm{C}$. Raman spectra show that, at $600^{\circ} \mathrm{C}$, the crystal damage has been repaired, which suggests that these remaining acceptor states are due to point defects or complexes.

\section{Acknowledgements}

The authors acknowledge the financial support of EPSRC (UK) in support of grant EP/E030130/1, and Royal society / RIA international joint project grant (2009-2011). J. Wasyluk would like to acknowledge the financial support of IRCSET and ICGEE Ireland, Postgraduate Award.

\section{References:}

[1] M. Bruel, Silicon on insulator material technology, Electron. Lett., 31 (1995) 1201-1202.

[2] H.S. Gamble, P.T. Baine, H. Wadsworth, Y.H. Low, P.V. Rainey, F.H. Ruddell, B.M. Armstrong, D.W. McNeill and S.J.N. Mitchell, Germanium on Sapphire, Int, Journal of High Speed Electronics and Systems, 18, (2008) 805-814.

[3] H.S. Gamble, B.M. Armstrong, P.T. Baine, Y.H. Low, P.V. Rainey, Y.W. Low, D.W. McNeill, S.J.N. Mitchell, J.H. Montgomery, and F.H. Ruddell, Germanium on sapphire substrates for system on a chip, Materials Science in Semiconductor Processing 11 (2008) 195-198.

[4] Information on http://www.solecon.com

[5] P. Rainey, J. Wasyluk, T. Perova, R. Hurley, N. Mitchell, D. McNeill, H. Gamble, and M. Armstrong, Micro-Raman and Spreading Resistance Analysis on Beveled Implanted Germanium for Layer Transfer Application, Electrochem. Solid State Lett. 14 (2011) H69-H72.

[6] Information on http://www.srim.org 
Gettering and Defect Engineering in Semiconductor Technology XIV doi:10.4028/www.scientific.net/SSP.178-179

Investigation of Germanium Implanted with Hydrogen for Layer Transfer Applications

doi:10.4028/www.scientific.net/SSP.178-179.295 